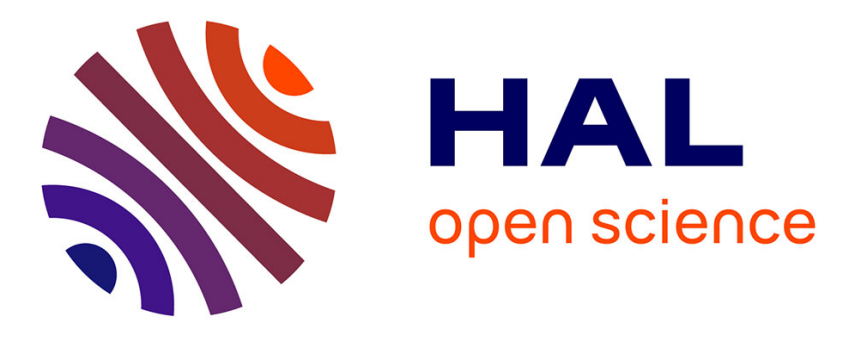

\title{
On Symbolic Approaches to Integro-Differential Equations
}

\author{
François Boulier, François Lemaire, Markus Rosenkranz, Rosane Ushirobira, \\ Nathalie Verdière
}

\section{- To cite this version:}

François Boulier, François Lemaire, Markus Rosenkranz, Rosane Ushirobira, Nathalie Verdière. On Symbolic Approaches to Integro-Differential Equations. Alban Quadrat; Eva Zerz. Algebraic and Symbolic Computation Methods in Dynamical Systems, 9, Springer, pp.161-182, 2020, Advances in Delays and Dynamics, 10.1007/978-3-030-38356-5_6 . hal-01367138v2

\section{HAL Id: hal-01367138 https://hal.science/hal-01367138v2}

Submitted on 18 Oct 2016

HAL is a multi-disciplinary open access archive for the deposit and dissemination of scientific research documents, whether they are published or not. The documents may come from teaching and research institutions in France or abroad, or from public or private research centers.
L'archive ouverte pluridisciplinaire HAL, est destinée au dépôt et à la diffusion de documents scientifiques de niveau recherche, publiés ou non, émanant des établissements d'enseignement et de recherche français ou étrangers, des laboratoires publics ou privés. 


\title{
On Symbolic Approaches to Integro-Differential Equations
}

François Boulier and François Lemaire and Markus Rosenkranz and Rosane Ushirobira and Nathalie Verdière

\begin{abstract}
Recent progress in computer algebra has opened new opportunities for the parameter estimation problem in nonlinear control theory, by means of integrodifferential input-output equations. This paper recalls the origin of integro-differential equations. It presents new opportunities in nonlinear control theory. Finally, it reviews related recent theoretical approaches on integro-differential algebras, illustrating what an integro-differential elimination method might be and what benefits the parameter estimation problem would gain from it.
\end{abstract}

\section{Introduction}

Under the impulse of the founding papers of Fliess [25], a school of researchers developed an approach of nonlinear control theory formulated within the framework of Ritt and Kolchin differential algebra [34, 48]. This approach led to various constructive methods which were implemented on computer algebra software dedicated for differential algebra $[10,11]$. In this context, the present paper is concerned with a parameter estimation method, which is connected to an algorithmic

François Boulier

Univ. Lille, CNRS, Centrale Lille, UMR 9189, CRIStAL, CFHP

e-mail: Francois.Boulier@univ-lillel.fr

François Lemaire

Univ. Lille, CNRS, Centrale Lille, UMR 9189, CRIStAL, CFHP

e-mail: Francois. Lemaire@univ-lillel.fr

Markus Rosenkranz

University of Kent, SMSAS, e-mail: M. Rosenkranz@kent.ac.uk

Rosane Ushirobira

Inria Lille Nord Europe, Non-A, e-mail: Rosane.Ushirobira@inria.fr

Nathalie Verdière

University Le Havre, LMAH, e-mail: Nathalie.Verdiereduniv-lehavre. fr 
structural identifiability test, based on the computation of the so-called input-output equation of the parametric nonlinear dynamical system under investigation [20,41]. Since numerical integration is often much less sensitive to noisy data than numerical differentiation, the parameter estimation step of this method provides more reliable estimates of parameters, by first transforming the input-output equation into an integro-differential equation. This idea has been tested on a range of examples $[21,22,26,40,58,59]$. This important transformation of nonlinear differential equations to integro-differential ones, which so far has required some human skill, can now be achieved algorithmically $[8,13]$.

Integral equations have other advantages as compared to differential ones. First, they permit to handle non smooth functions, in particular, piecewise constant inputs $[21,22,26]$. Second, they may naturally depend on initial conditions: a feature which may be important for the parameter estimation problem. In both cases, it may be interesting to bypass differential elimination in order to compute the desired equation.

These results and this research were at least partially motivated by purely theoretical studies of the algebraic properties of integro-differential algebras and their operator rings [3-5, 27, 29, 45,52]. In turn, they raise the fascinating and difficult task of extending the Ritt-Kolchin theory known as differential algebra to the broader theory integro-differential algebra since integral or integro-differential equations are not allowed within the framework of differential algebra. One important goal would be an elimination theory for integro-differential algebra. It would allow the computation of integro-differential input-output equations using a wider set of operations than in differential algebra, hence possibly faster computations as well as a greater variety of formulations for the input-output equations.

This paper is structured as follows. Section 2 recalls the origin of integrodifferential equations. Here, the term "origin" carries two meanings: what are the first historic examples of integro-differential models? and what kind of modelling processes lead to such models? This section will prove interesting for readers who discover integro-differential equations and for algebraists who are not aware of the needs of modellers. Section 3 sketches the application to parameter estimation for nonlinear dynamical systems that motivated this new interest for integro-differential equations. This section will be interesting for applied researchers who are not aware of some key properties of Ritt and Kolchin differential algebra: properties that need not generalize to the integro-differential framework. Last, Section 4 reviews some attempts to design algebraic theories of integro-differential equations and some of the many issues that need to be addressed. It illustrates also, via two examples, what an integro-differential elimination method could be and what would be the benefit to the parameter estimation problem. 


\section{Origin of Integro-Differential Models}

One of the simplest nonlinear integro-differential models studied in the literature is the Volterra-Kostitzin model [35, pages 66-69], which may be used for describing the evolution of a population, in a closed environment, intoxicated by its own metabolic products (other applications of the same model are considered in Kostitzin's book). It is an integro-differential equation since the unknown function $y(t)$ appears both differentiated and under some integral sign.

$$
\frac{\mathrm{d} y}{\mathrm{~d} t}(t)=\varepsilon y(t)-k y(t)^{2}-c y(t) \int_{t-T}^{t} K(t-\tau) y(\tau) \mathrm{d} \tau .
$$

The independent variable $t$ is time. The dependent variable $y(t)$ is the population, varying with time. The symbols $\varepsilon, k, c$ and $T$ denote parameters. The kernel (or nucleus) $K(t, \tau)=K(t-\tau)$ is the residual action function. For instance, it could be very similar to a "survival function" in population dynamics [31, page 3]: a decreasing function, starting at $K(0)=1$, equal to 0 outside the interval $[0, T]$. Then $K(t-\tau)$ would represent the "toxicity factor" of metabolic products which are the most toxic when produced, at $t=\tau$, become less toxic with the time, and have a negligible toxic effect at time $t=\tau+T$.

As we shall see later, nontrivial kernels introduce difficulties in the symbolic treatment of integro-differential equations. It is thus interesting to remark that a simplified version of the Volterra-Kostitzin model, with a trivial kernel $K(t, \tau)=$ 1 , was studied by Kostitzin himself (the model is then equivalent to a differential equation of order two). It was more recently reconsidered in [17] and [43, chapter 4] and fitted against experimental data, in order to validate its pertinence.

\subsection{Hereditary Theories}

In integral or integro-differential models, integral terms depend on kernels of the very special form $K(t, \tau)=K(t-\tau)$. Such kernels permit to express "hereditary", "historical" or "plastic" effects, i.e. the idea that the evolution of the current state of the system being modelled, depends not only on the current state but also on its past. The original qualifier is "hereditary". The qualifier "historical" was suggested by Volterra [62, page 300] to avoid any confusion with biological notions. The qualifier "plastic" (by opposition to "elastic") is used in structural mechanics [38, page 59].

\subsubsection{Historical Origin}

It is interesting to remark that biology is one of the first scientific domains where hereditary modelling was considered to be promising. In [60, page 295], Volterra claims to have coined the expression "integro-differential equation". A few lines 
further, he refers to an article by Picard [44], which contains the following paragraph (page 194), translated from French:

But heredity plays especially a major role in life sciences and we do not know if we will ever be able to use the mathematical tool for the intimate study of biological phenomena, and if we will not always need to restrict ourselves to rough averages and frequency curves. We should not, however, reduce in advance our mathematical conception of the world, and we can dream of functional equations more complicated than the former [differential] ones because they will involve, in addition, integrals taken between a very distant past and the present time, integrals which will bring their part of heredity.

The study of hereditary models is strongly connected with the theory of functionals, i.e. functions $z$ that depend on all the values that some other function $y(t)$ may take over some range $a \leq t \leq b$. This was investigated in detail by Volterra [63], who sketched hereditary formulations of magnetism, electricity, elasticity, ... It is intimately related to the theory of the convolution product, which arises in distribution theory [24], and whose algebraic properties were much studied by Volterra, as a special case of the "composition" of two functions. See [63] for the theory and [16, pages 293-294] for more on the history.

\subsection{Some Classical Integro-Differential Models}

\subsubsection{A Predator-Prey Model}

The following integro-differential system [62, pages 328-329] models two populations: one of them feeds on the other. It enhances a classical Volterra model of population dynamics. Volterra models here the fact that the increase of population does not depend only on the current amount of food available but also on the food which was available in the past.

$$
\begin{aligned}
& \frac{\mathrm{d} y_{1}}{\mathrm{~d} t}(t)=y_{1}(t)\left(\varepsilon_{1}-\gamma_{1} y_{2}(t)-\int_{0}^{t} f_{1}(t-\tau) y_{2}(\tau) \mathrm{d} \tau\right), \\
& \frac{\mathrm{d} y_{2}}{\mathrm{~d} t}(t)=y_{2}(t)\left(-\varepsilon_{2}+\gamma_{2} y_{1}(t)+\int_{0}^{t} f_{2}(t-\tau) y_{1}(\tau) \mathrm{d} \tau\right) .
\end{aligned}
$$

\subsubsection{Elastic Torsion of a Wire}

This example is borrowed from [63, chapter V, pages 147-149]. To a first approximation the connection between the moment $m$ of the torsional couple and the corresponding angle of torsion $\omega$ is given, in the case of static equilibrium, by the linear relation $\omega=k m$ where $k$ is a constant depending on the characteristics of the wire. Let $m(\tau)$ denote the torsional moment acting on the wire at time $\tau$. In order to find the angle of torsion $\omega(t)$ at time $t$ we must add to the right-hand side of $\omega(t)=k m(t)$ a corrective term depending on all the values of $m(\tau)$ for $\tau$ prior 
to $t$, and therefore a functional of $m(\tau)$. Assume the hereditary effects modelled by this functional is linear. One then obtains the following relation between $\omega(t)$ and $m(t)$ :

$$
\omega(t)=k m(t)+\int_{-\infty}^{t} f(t, \tau) m(\tau) \mathrm{d} \tau .
$$

Solving this Volterra integral equation [63, chapter II, page 44] with respect to $m(t)$, denoting the reciprocal kernel of $\frac{1}{k} f(t, \tau)$ by $\varphi(t, \tau)$ and assuming the hereditary effects prior to $t=0$ are negligible, we get

$$
m(t)=\frac{1}{k} \omega(t)+\int_{0}^{t} \varphi(t, \tau) \omega(\tau) \mathrm{d} \tau .
$$

Let us pass now from the static to the dynamic case and try to study the oscillations of the wire. For this, suppose that the angular velocity and acceleration are no longer negligible. The equation of motion of the wire is obtained from (3) by means of d'Alembert's principle, by substituting

$$
m(t)-\mu \frac{\mathrm{d}^{2} \omega}{\mathrm{d} t^{2}}(t) \quad(\mu \text { constant })
$$

for $m(t)$. We then get an integro-differential equation giving $\omega(t)$ in terms of $m(t)$ :

$$
m(t)-\mu \frac{\mathrm{d}^{2} \omega}{\mathrm{d} t^{2}}(t)=h \omega(t)+\int_{0}^{t} \varphi(t, \tau) \omega(\tau) \mathrm{d} \tau
$$

\subsubsection{Propagation of a Nervous Impulse}

This model is borrowed from [46, chapter XXXV, eq (32), page 426]. See also [31, chapter 1, page 5]. This nonpolynomial integral model is interesting because it has a trivial kernel and is equivalent to a polynomial system of integro-differential equations.

It is currently widely admitted that nervous impulses are propagated as follows in neurons: differences of ionic concentrations between the inside and the outside of axons make their membranes polarized. The occurence of an electric current in the neighborhood of some region of interest opens ionic channels, causing changes of ionic concentrations, hence an electric current in the region itself. The nervous influx is obtained by repeating this phenomenon along the whole axon. The details of the ionic activities, at a fixed position of the axon, are described by the famous Hodgkin-Huxley nonlinear differential model [33, chapter 5, pages 205-206].

The following model is probably older than the Hodgkin-Huxley model [30]. It is built on quite similar biological hypotheses and is concerned by the distance $u(t)$ traveled by an influx along a nerve (an axon, possibly). The parameter $I$ represents an electric current suddenly established in the neighborhood region at $t=0$. The 
parameter $h$ is a concentration threshold above which a nerve excitation is triggered [46, eq (6), page 379]. It is assumed that the ionic concentration and the electric current satisfy a linear differential equation of first order, depending on two parameters $K$ and $k$ [46, eq (18), page 423]. The time $t_{1}$ at which the region of interest releases an influx is then a function of $k, K, h$ and $I$. The parameter $\alpha$ is a constant depending on physical properties of the nerve: radius, specific resistances of the core of the nerve and its surrounding sheaths [46, eq (10), page 421]. The integral term of the model comes here from the fact that the distance is an integral of the speed [46, eq (21), page 424]. Since it is not motivated by any hereditary consideration, the absence of any nontrivial kernel is not surprising:

$$
h e^{\alpha u(t)+k t}=\frac{h K I}{K I-k h}+K I \int_{t_{1}}^{t} e^{\alpha u(\tau)+k \tau} \mathrm{d} \tau .
$$

Let now $v(t)$ be the exponential. The nonpolynomial integral equation (5) can be encoded by the following polynomial integro-differential system:

$$
\begin{aligned}
& \frac{\mathrm{d} v}{\mathrm{~d} t}(t)=\left(\alpha \frac{\mathrm{d} u}{\mathrm{~d} t}(t)+k\right) v(t), \\
& h v(t)=\frac{h K I}{K I-k h}+K I \int_{t_{1}}^{t} v(\tau) \mathrm{d} \tau .
\end{aligned}
$$

Of course, the equation (5) can also easily be transformed to differential form, by simple differentiations. However, this is no longer true when considering a timevarying (possibly non-smooth) current $I(t)$.

\section{Integro-Differential Equations for Parameter Estimation}

In this section, we present the application to parameters estimation for nonlinear dynamical systems that motivated our interest for integro-differential algebra. Together with the application, we introduce key concepts of differential algebra. In the next section, we will discuss issues raised by their generalization to integro-differential algebra.

\subsection{Statement of the Estimation Problem}

The academic two-compartment model depicted in Figure 1 is a close variant of [59, (1), page 517] endowed with an input $u(t)$. Compartment 1 represents the blood system and compartment 2 represents some organ. Both compartments are supposed to have unit volumes. The function $u(t)$, which has the dimension of a flow, represents a medical drug, injected in compartment 1 . The drug diffuses between the two 


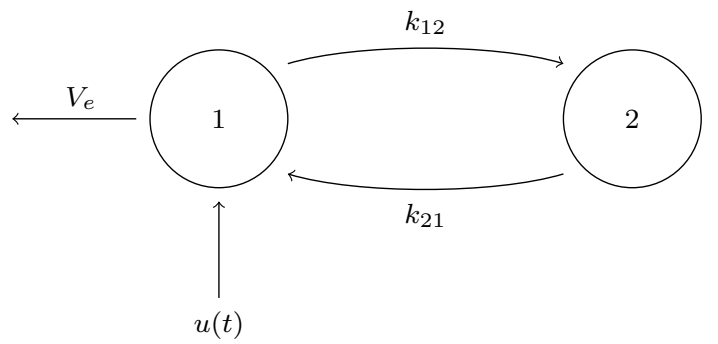

Fig. 1 A two-compartment model featuring three parameters.

compartments, following linear laws: the proportionality constants are named $k_{12}$ and $k_{21}$. The drug exits compartment 1 , following a law of Michaelis-Menten type. Such a law indicates a hidden enzymatic reaction. In general, it depends on two constants $V_{e}$ and $k_{e}$. For the sake of simplicity, it is assumed that $k_{e}=1$. The state variables in this system are $x_{1}(t)$ and $x_{2}(t)$. They represent the concentrations of drug in each compartment. This information is sufficient to write the two first equations of the mathematical model (7). The last equation of (7) states that the output, denoted $y(t)$, is equal to $x_{1}(t)$. This means that only $x_{1}(t)$ is observed: some numerical data are available for $x_{1}(t)$ but not for $x_{2}(t)$. The problem addressed here then consists in estimating the three parameters $k_{12}, k_{21}$ and $V_{e}$ from these data and the knowledge of $u(t)$.

$$
\begin{aligned}
& \dot{x}_{1}(t)=-k_{12} x_{1}(t)+k_{21} x_{2}(t)-\frac{V_{e} x_{1}(t)}{1+x_{1}(t)}+u(t), \\
& \dot{x}_{2}(t)=k_{12} x_{1}(t)-k_{21} x_{2}(t), \\
& y(t)=x_{1}(t) .
\end{aligned}
$$

\subsection{The Algebraic Setting}

\subsubsection{On the Solutions}

Ritt and Kolchin differential algebra provides an algebraic framework for polynomial differential systems. Differential systems involving rational fractions, such as (7), are easily handled. Other kinds of nonlinearities are not directly covered by the theory but many nonpolynomial systems can be transformed into polynomial ones, using techniques similar to the one we used for the propagation model of nervous impulses. Differential algebra imposes, however, another restriction, which is more important to us, since it reduces its applicability to control theory: the solu- 
tions of the systems under study are supposed to belong to integral domains (e.g. an equation such as $u(t) v(t)=0$ would imply that $u(t)=0$ or $v(t)=0$ ) and must be differentiable infinitely many times. The input $u(t)$ of (7) must then be smooth. One cannot study the case of a piecewise constant function $u(t)$ without leaving the realm of differential algebra.

\subsubsection{Differential Polynomial Ideal}

Subtract right-hand sides from left-hand sides of (7). Multiply the first equation by its denominator and state that this latter is nonzero. One obtains a system of three differential polynomial equations and one inequation:

$$
p_{1}=p_{2}=p_{3}=0, \quad 1+x_{1} \neq 0 .
$$

The left-hand sides of (8) belong to the differential polynomial ring

$$
\mathscr{R}=\mathbb{Q}\left(k_{12}, k_{21}, V_{e}\right)\left\{u, y, x_{1}, x_{2}\right\} .
$$

The three symbols $y, x_{1}, x_{2}$ are differential indeterminates. To this system, one associates a differential ideal

$$
\mathfrak{A}=\left[p_{1}, p_{2}, p_{3}\right]:\left(1+x_{1}\right)^{\infty} .
$$

Technically, the ideal $\mathfrak{A}$ is defined as the ideal of $\mathscr{R}$ generated by the three differential polynomials and their derivatives up to any order, saturated by the multiplicative family generated by $1+x_{1}$. This means that if any differential polynomial of the form $\left(1+x_{1}\right) g$ belongs to $\mathfrak{A}$, then $g$ itself belongs to $\mathfrak{A}$. It can be proved that $\mathfrak{A}$ is a prime (hence radical) differential ideal.

\subsubsection{Theorem of Zeros}

As already pointed out, in differential algebra, solutions are sought in differential rings that are free of zero-divisors. This restriction has some drawbacks for applications in control theory. Algebraically, it has a big advantage: the differential ideal $\mathfrak{A}$ is then the set of all differential polynomials that annihilate over the whole solution set of (7). In particular, a Theorem of Zeros [48, chapter I, 16] holds in differential algebra:

Theorem 1. A differential polynomial $g$ annihilates over all the solutions of a system of differential polynomial equations $f_{1}=f_{2}=\cdots=f_{n}=0$ if, and only if, a power of $g$ belongs to the differential ideal generated by $f_{1}, f_{2}, \ldots, f_{n}$.

The formulation above is not completely precise since the algebraic structure $\mathscr{S}$ which is supposed to contain the solutions is not given. Many precise variants could 
be given: $\mathscr{S}$ could be some differential field, an algebra of formal power series, a field of meromorphic functions ... See [9] for more details on this question.

\subsubsection{Elimination Theory}

An elimination theory has been available in differential algebra from its very beginning [48,57]. Some algorithms such as [39, diffgrob], [14, 15, RosenfeldGroebner], [47, rif], [2,36,49, Thomas algorithm] are implemented in computer algebra systems. In the sequel, we concentrate on RosenfeldGroebner and its most recent implementation in the MAPLE package [10, DifferentialAlgebra]. The first argument of such an algorithm is a system of differential polynomial equations and inequations. The second argument is a ranking, i.e. a total ordering on the set

$$
\left\{k_{12}, k_{21}, V_{e}\right\} \cup\left\{w^{(r)} \mid r \geq 0, w \text { differential indeterminate }\right\} .
$$

For an example such as (8), the output of the software is a regular differential chain - a notion slightly more general than Ritt's characteristic set [7, Definition 3.1] — of the differential ideal $\mathfrak{A}$ w.r.t. the ranking. Regular differential chains are finite sets of differential polynomials. By choosing a suitable ranking such as

(the derivatives of $\left.x_{1}, x_{2}\right) \gg$ (the derivatives of $\left.y, u\right) \gg$ (the parameters),

one can directly read in the output of the software the differential polynomial of $\mathfrak{A}$ which has the lowest rank w.r.t. the ranking. This differential polynomial is the so-called differential input-output equation of (7). It depends only on $y, u$, their derivatives, and the parameters to be estimated.

\subsection{The Input-Output Equation of the Problem}

A pretty-printed form of the differential input-output equation of (7) is:

$$
\begin{aligned}
-\theta_{1} u(t)+\theta_{2} \frac{y(t)}{y(t)+1} & +\theta_{3} \frac{\mathrm{d}}{\mathrm{d} t}\left(\frac{y(t)^{2}}{y(t)+1}\right) \\
& -\theta_{4} \frac{\mathrm{d}}{\mathrm{d} t}\left(\frac{1}{y(t)+1}\right)=\dot{u}(t)-\ddot{y}(t),
\end{aligned}
$$

where the $\theta_{i}$ stand for the following blocks of parameters:

$$
\theta_{1}=k_{21}, \quad \theta_{2}=k_{21} V_{e}, \quad \theta_{3}=k_{12}+k_{21}, \quad \theta_{4}=k_{12}+k_{21}+V_{e} .
$$




\subsubsection{Structural Identifiability Study}

The structural identifiability study is a preliminary study of the input-output equation. It can be viewed as a theoretical parameter estimation process, where the observed function $y(t)$, the input $u(t)$, and their derivatives up to any order are supposed to be as "generic" as possible and perfectly known.

A huge amount of literature is devoted to this question. See [1, 23, 37, 41, 42, 56, 59] and the references therein.

In our example, the structural identifiability study leads in a straightforward way to the desired conclusion of the global structural identifiability for this model. The essential argument is as follows:

1. Evaluating (9) at (at least) four different values of $t$, it is possible to build an invertible linear system whose unknowns are the blocks of parameters $\theta_{i}$.

2. Knowing the blocks of parameters $\theta_{i}$, it is easy to recover the values of the model parameters $k_{12}, k_{21}, V_{e}$, by solving the polynomial system (10).

It is worth noticing that step 1 of this argument eventually relies on the assumption that (9) is the equation of minimal order and degree constraining $y(t), u(t)$ and the parameters to be estimated. Ultimately, this argument relies on the Theorem of Zeros.

Indeed, on some other example, a non-minimal input-output equation could be artificially obtained by adding a polynomial of the form $\theta m(\theta$ any parameter block, $m$ belonging to the differential ideal $\mathfrak{A}$ ) to the minimal equation. At step 1 , such a polynomial $m$ would always evaluate to zero, yielding a linear system that would always be singular. The whole argument would then collapse.

\subsubsection{Integro-Differential Form of the Input-Output Equation}

A parameter estimation method can be designed by implementing "numerically" the steps 1 and 2 above, using the available numerical data for the observed function $y(t)$ and the input $u(t)$. If the data is noisy (but not only then), a straightforward implementation is however very likely to produce useless results since the second derivative of $y(t)$ then needs to be estimated numerically. In order to obtain more accurate results, it is desirable to convert the differential input-output equation (9) to integral form since numerical integration is less sensitive to noise than numerical differentiation. There are different ways to do it. Some methods are given in [12]. One possibility consists in applying twice the integration operator on (9). On our example, the result is the nonlinear Volterra integral equation (11). This formula still involves a derivative of the output, but evaluated at $t=a$. Viewing this derivative as an extra parameter $\theta_{5}$, to be estimated, Equation (11) does not involve any derivative of the output: 


$$
\begin{array}{r}
-\theta_{1} \int_{a}^{t} \int_{a}^{\tau_{1}} u\left(\tau_{2}\right) \mathrm{d} \tau_{2} \mathrm{~d} \tau_{1} \\
+\theta_{2} \int_{a}^{t} \int_{a}^{\tau_{1}} \frac{y\left(\tau_{2}\right)}{y\left(\tau_{2}\right)+1} \mathrm{~d} \tau_{2} \mathrm{~d} \tau_{1} \\
+\theta_{3}\left(\int_{a}^{t} \frac{y(\tau)^{2}}{y(\tau)+1} \mathrm{~d} \tau-\frac{y(a)^{2}}{y(a)+1}(t-a)\right) \\
-\theta_{4}\left(\int_{a}^{t} \frac{1}{y(\tau)+1} \mathrm{~d} \tau-\frac{1}{y(a)+1}(t-a)\right) \\
-\dot{y}(a)(t-a) \\
=\int_{a}^{t} u(\tau) \mathrm{d} \tau-u(a)(t-a)-y(t)+y(a) .
\end{array}
$$

In general, the resulting formula is an integro-differential equation. The method outlined here always produces formulas with trivial kernels. It can be completely automated, thanks to recent progresses in computer algebra $[8,12,13]$.

\subsubsection{Actual Parameter Estimation}

An integro-differential input-output equation such as (11) is used to build an overdetermined ${ }^{1}$ linear system, whose unknowns are the parameter blocks $\theta_{i}$. Its solutions, obtained by linear least squares, may be used as a first guess for nonlinear least squares such as the Levenberg-Marquardt method or Linear Matrix Inequalities. See $[19,20,41,59]$.

Recovering the initial model parameters from refined estimates of the parameter blocks $\theta_{i}$ is usually a difficult problem for which no satisfactory general solution is known. An important difficulty is raised by possible algebraic relations between blocks of parameters. In our example, we have such a relation:

$$
\theta_{1}\left(\theta_{3}-\theta_{4}\right)+\theta_{2}=0
$$

\subsection{Algorithmic Transformation to Integro-Differential Form}

Transforming (9) into the integral equation (11) is very easy because the former equation has the special form

$$
\sum \theta_{i} \frac{\mathrm{d}}{\mathrm{d} t} F_{i}
$$

where the $\theta_{i}$ are constant expressions (their derivatives are zero) and the $F_{i}$ are order zero fractions. However, the input-output equations returned by differential

\footnotetext{
${ }^{1}$ A square linear system is sufficient for the identifiability study, which is of theoretical nature. On real, possibly noisy, data, it is preferable to evaluate the input-output equations at much more values of $t$, and thereby obtain an overdetermined linear system.
} 
elimination algorithms do not always have this shape. Instead, they have the form of polynomials in the derivatives of the differential indeterminates, which implies that the $\frac{\mathrm{d}}{\mathrm{d} t} F_{i}$ expressions are expanded. A first algorithm for converting this raw form into (9) is published in [8], with a flaw fixed in [12]. An enhanced version, with a canonical output, is published in [13].

\section{Towards Algebraic Theories}

The systematic treatment of integral operators in algebra-usually under the name of Rota-Baxter operators-has been inaugurated with Glen Baxter's seminal paper [6]. While originally viewed in a probability context, Rota-Baxter operators have soon found interest in broader areas of algebra, especially through Gian-Carlo Rota's well-known papers $[54,55]$. For a modern survey on Rota-Baxter algebra we refer to the monograph [28].

The notion of Rota-Baxter operator was combined with differential algebra structures in $[50,53]$ for creating an algebraic framework that allows a constructive treatment of boundary problems for linear ordinary differential equations. In particular, the Green's operator (resolvent operator), which maps the forcing function to the solution of the boundary problem, is expressed as a Fredholm integral operator that belongs to a suitable operator ring.

Let us explain this in some more detail. We start from an integro-differential algebra $\left(\mathcal{F}, \partial, \int\right)$, meaning an algebra over some field $K$ with two $K$-linear operators $\partial, \int: \mathcal{F} \rightarrow \mathcal{F}$ that are supposed to capture differentiation and integration in an algebraic context. Hence the derivation $\partial$ is required to satisfy the Leibniz axiom (= product rule for differentiation) while $\int$ must satisfy the Rota-Baxter axiom (= integration by parts); moreover we stipulate $\partial \circ \int=1_{\mathcal{F}}$ for tying the two notions together, just as the fundamental theorem of calculus does in the case $\mathcal{F}=C^{\infty}(\mathbb{R})$; note that this is a special case of the generalized Leibniz integral rule (14). It turns out that the other composition is not quite the identity but $\int \circ \partial=1_{\mathcal{F}}-E$, where $E: \mathcal{F} \rightarrow \mathcal{F}$ is a multiplicative linear map that may be thought as the evaluation at the initialization point of the integral operator $\int$. Indeed, this is what happens in the most important example $\mathcal{F}=C^{\infty}(\mathbb{R})$ where $\partial f(x)=d f / d x$ and $\int f(x)=\int_{a}^{x} f(\xi) d \xi$ for some initialization point $a \in \mathbb{R}$; consequently, here $E: \mathcal{F} \rightarrow \mathbb{R}$ is the evaluation $f(x) \mapsto f(a)$. Of course $C^{\infty}(\mathbb{R})$ contains many other integro-differential algebras, for example the polynomials $\mathbb{R}[x]$ or the analytic functions $C^{\omega}(\mathbb{R})$, and various intermediate algebras like the exponential polynomials (real or complex linear combinations of $x^{k} e^{\lambda x}$ for any $k \in \mathbb{N}$ and $\lambda \in \mathbb{R}$ ).

Each integro-differential algebra $\left(\mathcal{F}, \partial, \int\right)$ now gives rise to an operator ring $\mathcal{F}\left[\partial, \int\right]$ that contains both differential operators such as $a(x) \partial^{2}+b(x) \partial+c(x)$ for coefficient functions $a(x), b(x), c(x) \in \mathcal{F}$ and integral operators such as $x^{2} e^{x} \int e^{-2 x}$, as well as evaluation operators $E_{a}$ for various points $a \in \mathbb{R}$. Note that the integral operator $x^{2} e^{x} \int e^{-2 x}$ is here understood as a non-commutative operator composition, acting as $f(x) \mapsto \int_{a}^{x} x^{2} e^{x-2 \xi} f(\xi)$. We refer to $\mathcal{F}\left[\partial, \int\right]$ as the integro-differential 
operator ring over $\mathcal{F}$. One can prove that every boundary problem over $\mathcal{F}$ has a Green's operator $G \in \mathcal{F}\left[\partial, \int\right]$, which can be determined algorithmically if one has a fundamental system of solutions for the underlying homogeneous differential equation [53, Thm. 26].

The treatment of linear partial differential equations is considerably more difficult. Of course one will replace $\left(\mathcal{F}, \partial, \int\right)$ by a structure $\left(\mathcal{F}, \partial_{x}, \partial_{y}, \int^{x}, \int^{y}\right)$, where $\mathcal{F}$ is an algebraic structure describing multivariate (for simplicity here: bivariate) functions with two derivations $\partial_{x}, \partial_{y}$ and two Rota-Baxter operators $\int^{x}, \int^{y}$. However, this would not lead us very far since even very simple examples like $u_{x}-$ $u_{y}=f$ cannot be solved in terms of these basic building blocks. One crucial missing piece is a structure for substitutions. For treating linear partial differential equations, it is sufficient to allow only linear substitutions like $u(x, y) \mapsto u(a x+b y, c x+d y)$ for $a, b, c, d \in \mathbb{R}$. The resulting algebraic structure, a so-called Rota-Baxter hierarchy, is surprisingly complex and has been described in [51]. In fact, the current setup omits the derivations $\partial_{x}, \partial_{y}$ for keeping the complications to a minimum (derivations are comparatively easy to add since their algebraic relations are far less complex than those connected with the Rota-Baxter operators).

Similar to the case of plain integro-differential algebras, every Rota-Baxter hierarchy comes with a multivariate operator ring that we could provisionally denote by $\mathcal{F}\left[\int^{x}, \int^{y}\right]$ or by $\mathcal{F}\left[\partial_{x}, \partial_{y}, \int^{x}, \int^{y}\right]$ if the derivations are added in. The main innovation from the ordinary case is that substitution operators $M^{*}=\left(\begin{array}{ll}a & b \\ c & d\end{array}\right)^{*}$, acting as described above for a matrix $M \in \mathbb{R}^{2 \times 2}$, are also part of the basic building blocks. Their interaction with the Rota-Baxter operators is complicated, but normal forms have been deduced [51, Thm 4.10], for the case of arbitrarily many variables.

Describing the nature of those operator relations would lead us too far afield for the present paper. It will suffice to mention just one special case of Axiom (7) of [51, Def. 2.3], namely

$$
\int^{x} M^{*} \int^{x}=M^{*} \int^{x} \int^{y}-\int^{x} M^{*} \int^{y}
$$

where $M \in \mathbb{R}^{2 \times 2}$ is the substitution matrix with $a=c=1, b=d=0$, acting by $u(x, y) \mapsto u(x, x)$. Written in the usual notation, this is exactly Dirichlet's rule to be mentioned in Section 4.1.3. However, the great advantage of the operator-ring framework is that there is a normal form (namely the right-hand side). In fact, we are confident that these normal forms are in fact canonical (meaning every simplification can only lead to a single normal form), which is equivalent to the algebraic statement that the chosen identities are a non-commutative Gröbner basis for the ideal of operator relations. This is work in progress, more than half of the proof is completed but there the derivations are very long.

Up to now we have spoken about linear differential and integral equations, both ordinary and partial (of course this includes also the mixed integro-differential cases). For passing to nonlinear equations, one can pass to the ring of integrodifferential polynomials. In the univariate case, this has been introduced in [52]. Essentially the same structure-but extended to the partial case as well as differential fractions-was subsequently treated in $[8,13]$. The basic idea in all case is that 
nested integrals like $\int u^{2} u^{\prime 3} \int u^{\prime} u^{\prime 2} \int u^{\prime \prime}\left(u^{\prime \prime \prime}\right)^{5}$ or $\int u^{\prime 2} \int u^{\prime \prime} \int u u^{\prime \prime 4}$, or linear combinations of these, are in canonical form only if the highest derivative of $u$ appears nonlinearly in each of the nested integrands. Hence the first example above is canonical, but the second is not.

While the algebraic investigation of the linear case (univariate and multivariate integro-differential operator rings) are now gradually maturing to some extent, the nonlinear case of integro-differential polynomials/fractions is wide open. In the last two sections we will only address two prominent issues that appear to pose considerable difficulties and, by the same token, a highly interesting arena of algebraic research.

\subsection{Computational Issues}

\subsubsection{The Generalized Leibniz Integral Rule}

Though there does exist applications of integro-differential equations which only need trivial kernels, this is certainly not the case of equations arising from hereditary modelling, which have the form:

$$
\int_{a}^{t} K(t, \tau) f(\tau) \mathrm{d} \tau
$$

Over such expressions, the formula

$$
\frac{\mathrm{d}}{\mathrm{d} t} \int_{a}^{t}=\text { the identity operator }
$$

does not hold anymore. Instead, one must apply the general form of Leibniz integral rule, which gives:

$$
\frac{\mathrm{d}}{\mathrm{d} t} \int_{a}^{t} K(t, \tau) f(\tau) \mathrm{d} \tau=\int_{a}^{t} \frac{\mathrm{d} K}{\mathrm{~d} t}(t, \tau) f(\tau) \mathrm{d} \tau+K(t, t) f(t) .
$$

However, this formula raises a computational problem, in the case of singular kernels. Let us quote Volterra [63, chapter II, page 54]:

It is rather curious to note that it was precisely these singular cases that were the first in order of time to arise; the first integral equation considered goes back to Abel and is as follows:

$$
\sqrt{2 g} z(t)=\int_{0}^{t} \frac{y(\tau)}{(t-\tau)^{\frac{1}{2}}} \mathrm{~d} \tau
$$

and the kernel $(t-\tau)^{-\frac{1}{2}}$ becomes infinite at $\tau=t$. 
Differentiating (15) causes a division by zero. In particular, we see that, contrarily to what happens in differential algebra, differentiation of integro-differential expressions is not always defined.

\subsubsection{Integration by Change of Variable}

The change of variable formula is:

$$
\int_{a}^{b} F(\varphi(t)) \frac{\mathrm{d} \varphi}{\mathrm{d} t}(t) \mathrm{d} t=\int_{\varphi(a)}^{\varphi(b)} F(t) \mathrm{d} t .
$$

Kostitzin applies it [35, page 68] for studying the Volterra-Kostitzin model in the case of a trivial kernel. Having proven that

$$
\frac{\mathrm{d} y}{\mathrm{~d} t}(t)=F(y(t))
$$

he deduces that

$$
t=\int_{y(0)}^{y(t)} \frac{1}{F(\tau)} \mathrm{d} \tau .
$$

This identity shows that, in an integro-differential algebra theory in which integration operators with general bounds would be allowed, the equality test between two expressions might be a difficult problem.

\subsubsection{Dirichlet's Rule}

Volterra attributes this formula to Dirichlet in [61, page 36] and often uses it:

$$
\int_{a}^{t} \int_{a}^{\tau} F\left(\tau_{2}, \tau\right) \mathrm{d} \tau_{2} \mathrm{~d} \tau=\int_{a}^{t} \int_{\tau_{2}}^{t} F\left(\tau_{2}, \tau\right) \mathrm{d} \tau \mathrm{d} \tau_{2} .
$$

In the parameter estimation problem described in Section 3, this formula could have been used in order to produce another form of the integral equation (11), which would have involved a non trivial kernel. Indeed:

$$
\int_{a}^{t} \int_{a}^{\tau} F\left(\tau_{2}\right) \mathrm{d} \tau_{2} \mathrm{~d} \tau=\int_{a}^{t}(t-\tau) F(\tau) \mathrm{d} \tau .
$$

This formula illustrates another difficulty that arises when testing equality between two integro-differential expressions. Recent progresses on this issue are given in [51]. 


\subsection{On Generalizations of the Theorem of Zeros}

As pointed out in Section 3, the Theorem of Zeros is implicitly used in the structural identifiability test based on the input-output equation. This section shows that it might not generalize in the integro-differential framework. Observe a similar difficulty occurs in other theories such as difference algebra [18].

Let us define an integro-differential ring $\mathscr{R}=\mathbb{Q}\{u\}$ as the smallest ring containing the integro-differential indeterminate $u$, the rational numbers, stable under derivation and integration. For simplicity, let us restrict ourselves to the case of a derivation $\delta$ being the left inverse of the integration $\int$, i.e. an abstract form of (13). Let us denote $x=\int 1$.

Given any $p \in \mathscr{R}$, let us define the integro-differential ideal generated by $p$ as the smallest ideal of the ring $\mathscr{R}$, containing $p$, stable under derivation and integration. Let us denote it $[p]$. Consider now

$$
p=u-\int u,
$$

which is meant to be an abstract form of the left-hand side of the integral equation

$$
u(t)-\int_{0}^{t} u(\tau) \mathrm{d} \tau=0 .
$$

This equation admits $u(t)=0$ for unique solution. However, we prove below that $u^{m} \notin[p]$ for any non-negative integer $m$, i.e. that, in the algebraic framework sketched above, the Theorem of Zeros does not hold.

Proposition 1. Take $p \in \mathscr{R}$. Then, any element $q$ of $[p]$ can be written as $q=$ $\sum_{i=1}^{s} a_{i} M_{i}$ where $a_{i} \in \mathbb{Q}$ and each $M_{i}$ has the form

$$
M_{i}=m_{i, 0} \int m_{i, 1} \int \cdots \int m_{i, k-1} \int m_{i, k}\left(\delta^{b_{i}} p\right) \int m_{i, k+1} \int \cdots \int m_{i, t_{i}},
$$

where $b_{i}$ is a non-negative integer, the $m_{i, j}$ are monomials in $x$, and $u$ and its derivatives.

Proof. Admitted.

Let us denote by $w\left(M_{i}\right)$ the weight in $u$ of any $M_{i}$ of the form of (17), with $w\left(M_{i}\right)=1+\sum_{j=0}^{t_{i}} \operatorname{deg}\left(m_{i, j},[u, \delta u, \ldots]\right)$, where $\operatorname{deg}\left(m_{i, j},[u, \delta u, \ldots]\right)$ denotes the total degree of $m_{i, j}$ in the variables $u, \delta u, \ldots$

Lemma 1. Take $p=u-\int u$ and consider some $M_{i}$ in the form of (17). Then replacing $u$ by $\alpha$ in $M_{i}$ yields $\alpha^{w\left(M_{i}\right)} M_{i}$, for any $\alpha \in \mathbb{Q}$. Moreover, replacing $u$ by $e^{x}$ in $M_{i}$ yields 0 if $b_{i}>0$, or a polynomial in $x$ and $e^{x}$ whose degree in $e^{x}$ is at most $w\left(M_{i}\right)-1$ otherwise.

Proof. Immediate. 
Proposition 2. Take $p=u-\int u$. Then $u^{m} \notin[p]$ for any non-negative integer $m$.

Proof. Assume that $u^{m} \in[p]$ for some $m$. Let us prove that this yields a contradiction. By Proposition 1, we have $u^{m}=\sum_{i=1}^{s} a_{i} M_{i}$ where the $a_{i}$ are in $\mathbb{Q}$ and the $M_{i}$ have the form of (17). The monomial $u^{m}$ is homogeneous of degree $m$. By an homogeneity argument and by Lemma 1 , all $M_{i}$ have the same weights $w\left(M_{i}\right)=m$ in $u$.

Substituting $u=e^{x}$ in $u^{m}$ yields $e^{m x}$. However, substituting $u=e^{x}$ in any $M_{i}$ either yields 0 if $b_{i}>0$, or a polynomial in $x$ and $e^{x}$ whose degree in $e^{x}$ is at most $m-1$ by Lemma 1 . This yields a contradiction since $e^{x}, e^{2 x}, \ldots, e^{m x}$ are linearly independent over $\mathbb{Q}[x]$.

\subsection{On Derivation-Free Elimination}

The most challenging theoretical issue would consist in developing an elimination theory for integro-differential equations that would permit to bypass differential algebra methods. To our knowledge, such a derivation-free elimination theory has not been considered yet. To illustrate what it could ideally do, we show that Equation (11) can be obtained from Equation (7) without performing any differentiation. Let us slighty rewrite Equation (7) as

$$
\begin{aligned}
& f_{1}(t):=-\dot{y}(t)-k_{12} y(t)+k_{21} x_{2}(t)-V_{e} \frac{y(t)}{1+y(t)}+u(t), \\
& f_{2}(t):=-\dot{x}_{2}(t)+k_{12} y(t)-k_{21} x_{2}(t) .
\end{aligned}
$$

Since $f_{1}(t)$ and $f_{2}(t)$ are identically zero, we obtain the equation

$$
0=k_{21} \int_{a}^{t} \int_{a}^{\tau} f_{1}\left(\tau_{2}\right)+f_{2}\left(\tau_{2}\right) \mathrm{d} \tau_{2} \mathrm{~d} \tau+\int_{a}^{t} f_{1}(\tau) \mathrm{d} \tau
$$

Simplifying the previous equation yields

$$
\begin{array}{r}
k_{21} \int_{a}^{t} \int_{a}^{\tau} u\left(\tau_{2}\right) \mathrm{d} \tau_{2} \mathrm{~d} \tau-k_{21} V_{e} \int_{a}^{t} \int_{a}^{\tau} \frac{y\left(\tau_{2}\right)}{1+y\left(\tau_{2}\right)} \mathrm{d} \tau_{2} \mathrm{~d} \tau \\
-\left(k_{21}+k_{12}\right) \int_{a}^{t} y(\tau) \mathrm{d} \tau-V_{e} \int_{a}^{t} \frac{y(\tau)}{1+y(\tau)} \mathrm{d} \tau+k_{21}\left(y(a)+x_{2}(a)\right)(t-a) \\
=y(t)-y(a)-\int_{a}^{t} u(\tau) \mathrm{d} \tau
\end{array}
$$

From $f_{1}(a)=0$, one has

$$
k_{21} x_{2}(a)=\dot{y}(a)+k_{12} y(a)+V_{e} \frac{y(a)}{1+y(a)}-u(a) .
$$

Replace $k_{21} x_{2}(a)$ by its value in the last equation. Simple computations show that 


$$
\begin{array}{r}
k_{21}\left(\int_{a}^{t} \int_{a}^{\tau} u\left(\tau_{2}\right) \mathrm{d} \tau_{2} \mathrm{~d} \tau+y(a)(t-a)-\int_{a}^{t} y(\tau) \mathrm{d} \tau\right) \\
-k_{21} V_{e} \int_{a}^{t} \int_{a}^{\tau} \frac{y\left(\tau_{2}\right)}{1+y\left(\tau_{2}\right)} \mathrm{d} \tau_{2} \mathrm{~d} \tau-V_{e}\left(\int_{a}^{t} \frac{y(\tau)}{1+y(\tau)} \mathrm{d} \tau-\frac{y(a)}{1+y(a)}\right) \\
+k_{12}\left(y(a)(t-a)-\int_{a}^{t} y(\tau) \mathrm{d} \tau\right)+\dot{y}(a)(t-a) \\
=y(t)-y(a)-\int_{a}^{t} u(\tau) \mathrm{d} \tau+u(a)(t-a) .
\end{array}
$$

This last equation is equivalent (up to the sign) to (11), by using the properties $y^{2} /(1+y)=y-1+1 /(1+y)$ and $1 /(y+1)=1-y /(y+1)$.

\subsection{On Alternative Input-Output Equations}

A structural identifiability study, based on the differential input-output equation, was sketched in Section 3.3.1. Since this equation is computed in the strict framework of differential algebra, it does not feature any initial value of any non-observed variable. In some cases, however, the initial conditions of some non-observed variables are known, and their knowledge is necessary to prove the structural identifiability of the model. A first approach to overcome this difficulty is presented in [21,22]: the integration of the input-output equations followed by some further manipulations yields expressions involving the initial conditions of the non-observed variables, permitting to prove the structural identifiability. We show in this section that integrodifferential elimination offers another approach since it permits to compute integral input-output equations featuring naturally these important initial values. The system $\Sigma$ under study is inspired from [32]:

$$
\begin{aligned}
& \dot{x}_{1}(t)=\theta_{1} x_{2}(t)+u(t), \\
& \dot{x}_{2}(t)=\theta_{2} x_{1}(t) x_{2}(t)+\theta_{3} x_{2}(t)+u(t),
\end{aligned}
$$

together with the assumptions

- $x_{1}(0)=x_{10}$ is known,

- $x_{2}(t)$ and $u(t)$ are observed on some interval $\left[0, t_{0}\right]$,

- $x_{1}(t)$ is not observed on $\left.] 0, t_{0}\right]$.

The parameters to be estimated are $\theta_{1}, \theta_{2}$ and $\theta_{3}$. Differential elimination methods permit to compute the following differential input-output equation:

$$
\ddot{x}_{2}(t) x_{2}(t)-\dot{x}_{2}^{2}(t)+\dot{x}_{2}(t) u(t)-x_{2}(t) \dot{u}(t)-\theta_{1} \theta_{2} x_{2}^{3}(t)-\theta_{2} u(t) x_{2}^{2}(t) .
$$

The structural identifiability study sketched in Section 3.3.1 would conclude to the non-identifiability of $\Sigma$ since $\theta_{3}$ does not even appear in this equation. Converting 
this equation to integro-differential form would obviously not change this conclusion. Now, it is interesting to observe that one can obtain an expression depending on $\theta_{3}$ by evaluating (19) at $t=0$, provided that $x_{2}(0) \neq 0$. Another expression can be obtained if $x_{2}(0)=0$ and $\dot{x}_{2}(0) \neq 0$ : differentiating (19) and rewriting the term $\dot{x_{1}}(t)$ using (18) yields

$$
\ddot{x}_{2}(t)=\theta_{2}\left(\left(\theta_{1} x_{2}(t)+u(t)\right) x_{2}(t)+x_{1}(t) \dot{x}_{2}(t)\right)+\theta_{3} \dot{x}_{2}(t)+\dot{u}(t) .
$$

Evaluating this expression at $t=0$ provides an expression for $\theta_{3}$ as a function of $x_{1}(0), x_{2}(0), \dot{x_{2}}(0), \ddot{x}_{2}(0), u(0), \dot{u}(0), \theta_{1}$ and $\theta_{2}$. More generally, a formula can be obtained provided that some derivative of $x_{2}(t)$ does not vanish at $t=0$.

Let us now compute an integral input-output equation, using the integration operator from the beginning. First put $\Sigma$ in an integral form:

$$
\begin{aligned}
& x_{1}(t)=x_{10}+\int_{0}^{t} \theta_{1} x_{2}(\tau)+u(\tau) \mathrm{d} \tau, \\
& x_{2}(t)=x_{20}+\int_{0}^{t} \theta_{2} x_{1}(\tau) x_{2}(\tau)+\theta_{3} x_{2}(\tau)+u(\tau) \mathrm{d} \tau .
\end{aligned}
$$

Using (21) for replacing $x_{1}(t)$ by its value in (22) yields

$$
x_{2}(t)=x_{20}+\int_{0}^{t} \theta_{2}\left(x_{10}+\int_{0}^{\tau} \theta_{1} x_{2}\left(\tau_{2}\right)+u\left(\tau_{2}\right) \mathrm{d} \tau_{2}\right) x_{2}(\tau)
$$

Expanding (23) yields

$$
x_{2}(t)=I_{0}(t)+\left(x_{10} \theta_{2}+\theta_{3}\right) I_{1}(t)+\theta_{2} I_{2}(t)+\theta_{1} \theta_{2} I_{3}(t)
$$

with

$$
\begin{array}{ll}
I_{0}(t)=x_{20}+\int_{0}^{t} u(\tau) \mathrm{d} \tau, & I_{1}(t)=\int_{0}^{t} x_{2}(\tau) \mathrm{d} \tau \\
I_{2}(t)=\int_{0}^{t} x_{2}(\tau) \int_{0}^{\tau} u\left(\tau_{2}\right) \mathrm{d} \tau_{2} \mathrm{~d} \tau, & I_{3}(t)=\int_{0}^{t} x_{2}(\tau) \int_{0}^{\tau} x_{2}\left(\tau_{2}\right) \mathrm{d} \tau_{2} \mathrm{~d} \tau .
\end{array}
$$

Let us now follow the structural identifiability study sketched in Section 3.3.1 on (24). The linear system considered at step 1 is invertible since the three terms $I_{1}(t), I_{2}(t)$ and $I_{3}(t)$ are linearly independent ${ }^{2}$. Thus the structural identifiability study would infer the global structural identifiability of $\Sigma$. The input-output equa-

\footnotetext{
${ }^{2}$ Indeed, if the three terms were linearly dependent, there would exist three constants $A, B, C$ such that $A I_{1}(t)+B I_{2}(t)+C I_{3}(t)=0$ modulo the prime differential ideal $\mathfrak{A}$ generated by $\Sigma$. Differentiate this equation. Divide it by $x_{2}(t)$. Differentiate again. One gets $B u(t)+C x_{2}(t)=0$. However, $B u(t)+C x_{2}(t)$ is not reduced to zero by $\Sigma$, viewed as a regular differential chain (or a characteristic set) of $\mathfrak{A}$. This contradiction proves the linear independence of the three terms.
} 
tion obtained by integro-differential elimination is therefore not equivalent to the one obtained by plain differential elimination.

\section{References}

1. Stefania Audoly, Giuseppina Bellu, Leontina D'Angio, Maria Pia Saccomani, and Claudio Cobelli. Global Identifiability of Nonlinear Models of Biological Systems. IEEE Transactions on Biomedical Engineering, 48(1):55-65, 2001.

2. Thomas Bächler, Vladimir Gerdt, Markus Lange-Hegermann, and Daniel Robertz. Algorithmic Thomas Decomposition of Algebraic and Differential Systems. Journal of Symbolic Computation, 47(10):1233-1266, 2012.

3. Vladimir V. Bavula. The algebra of integro-differential operators on a polynomial algebra. $J$. Lond. Math. Soc., 83(2):517-543, 2011.

4. Vladimir V. Bavula. The algebra of integro-differential operators on an affine line and its modules. Journal of Pure and Applied Algebra, 17(3):495-529, 2013.

5. Vladimir V. Bavula. The algebra of polynomial integro-differential operators is a holonomic bimodule over the subalgebra of polynomial differential operators. Algebras and Representation Theory, 17(1):275-288, 2014.

6. Glen Baxter. An Operator Identity. Pacific J. Math., 8:649-663, 1958.

7. François Boulier and François Lemaire. A Normal Form Algorithm for Regular Differential Chains. Mathematics in Computer Science, 4(2):185-201, 2010. 10.1007/s11786-010-00603.

8. François Boulier, François Lemaire, Georg Regensburger, and Markus Rosenkranz. On the Integration of Differential Fractions. In Proceedings of the 38th international symposium on International symposium on symbolic and algebraic computation, ISSAC'13, pages 101-108, New York, NY, USA, 2013. ACM.

9. François Boulier and François Lemaire. A computer scientist point of view on Hilbert's differential theorem of zeros. (preprint), 2007. http://hal.archives-ouvertes. fr/ hal-00170091.

10. François Boulier and Edgardo Cheb-Terrab. DifferentialAlgebra. Package of MapleSoft MAPLE standard library since MAPLE 14, 2008.

11. François Boulier and Évelyne Hubert. diffalg. Package of MapleSoft MAPLE standard library from MAPLE V to MAPLE 13, 1996.

12. François Boulier, Anja Korporal, François Lemaire, Wilfrid Perruquetti, Adrien Poteaux, and Rosane Ushirobira. An Algorithm for Converting Nonlinear Differential Equations to Integral Equations with an Application to Parameter Estimation from Noisy Data. In LNCS 8660: Proceedings of Computer Algebra and Scientific Computing (CASC) 2014, pages 28-43, Warsaw, Poland, 2014.

13. François Boulier, Joseph Lallemand, François Lemaire, Georg Regensburger, and Markus Rosenkranz. Additive Normal Forms and Integration of Differential Fractions. Journal of Symbolic Computation, 77:16-38, 2016.

14. François Boulier, Daniel Lazard, François Ollivier, and Michel Petitot. Representation for the radical of a finitely generated differential ideal. In ISSAC'95: Proceedings of the 1995 international symposium on Symbolic and algebraic computation, pages 158-166, New York, NY, USA, 1995. ACM Press. http: //hal .archives-ouvertes.fr/hal-0 0138020.

15. François Boulier, Daniel Lazard, François Ollivier, and Michel Petitot. Computing representations for radicals of finitely generated differential ideals. Applicable Algebra in Engineering, Communication and Computing, 20(1):73-121, 2009. (1997 Techrep. IT306 of the LIFL).

16. Nicolas Bourbaki. Éléments d'Histoire des Mathématiques, volume iv of Collection Histoire de la Pensée. Hermann, second edition, 1969.

17. J. L. Chassé, J. M. Legay, and A. Pavé. Le modèle de Volterra-Kostitzin en dynamique des populations. Ajustement et interprétation des paramètres. Ann. Zool. Ecol. anim., 9(3), 1977. 
18. Richard Cohn. Difference Algebra. Interscience Publishers, 1965.

19. Lilianne Denis-Vidal, Zohra Cherfi, Vincent Talon, and El Hassane Brahmi. Parameter Identifiability and Parameter Estimation of a Diesel Engine Combustion Model. Journal of Applied Mathematics and Physics, 2:131-137, 2014.

20. Lilianne Denis-Vidal, Ghislaine Joly-Blanchard, and Céline Noiret. System identifiability (symbolic computation) and parameter estimation (numerical computation). In Numerical Algorithms, volume 34, pages 282-292, 2003.

21. Lilianne Denis-Vidal, Ghislaine Joly-Blanchard, and Nathalie Verdière. Identifiability of Ordinary or Delayed Nonlinear Models: a Distribution Approach. In proceedings of the IEEE, Munich, 2006.

22. Lilianne Denis-Vidal, Ghislaine Joly-Blanchard, and Nathalie Verdière. Identifiability and Estimation of Nonlinear Models: a Distribution Framework. In proceedings of the ECC, Greece, Koos, 2007.

23. Sette Diop and Michel Fliess. Nonlinear observability, identifiability, and persistent trajectories. In Proc. 30th CDC, pages 714-719, Brighton, 1991.

24. J. J. Duistermaat and J. A. C. Kolk. Distributions: Theory and Applications. Birkhäuser, 2010.

25. Michel Fliess. Automatique et corps différentiels. Forum Math., 1:227-238, 1989.

26. Michel Fliess, Mamadou Mboup, Hugues Mounier, and Hebertt Sira-Ramirez. Questioning some paradigms of signal processing via concrete examples. In G. Silva-Navarro H. SiraRamirez, editor, Algebraic Methods in Flatness, Signal Processing and State Estimation, Algebraic Methods in Flatness, Signal Processing and State Estimation, pages 1-21, Mexico, Nov 2003. Editorial Lagares.

27. Xing Gao and Li Guo. Constructions of Free Commutative Integro-Differential Algebras. In M. Barkatou, T. Cluzeau, G. Regensburger, and M. Rosenkranz, editors, Algebraic and Algorithmic Aspects of Differential and Integral Operators, volume 8372 of LNCS, pages 122, 2014.

28. Li Guo. An Introduction to Rota-Baxter Algebras. International Press, 2012.

29. Li Guo, Georg Regensburger, and Markus Rosenkranz. On integro-differential algebras. Journal of Pure and Applied Algebra, 218(3):456-473, 2014.

30. Alan Lloyd Hodgkin and Andrew F. Huxley. A Quantitative Description of Membrane Current and its Application to Conduction and Excitation in Nerve. J. Physiolog., 117:500-544, 1952.

31. Abdul J. Jerri. Introduction to Integral Equations with Applications, volume 93 of Monographs and Textbooks in Pure and Applied Mathematics. Marcel Dekker Inc., 1985.

32. Ghislaine Joly-Blanchard and Lilianne Denis-Vidal. Some remarks about an identifiability result of nonlinear systems. Automatica, 34(9):1151-1152, 1998.

33. James Keener and James Sneyd. Mathematical Physiology I: Cellular Physiology, volume 8/I of Interdisciplinary Applied Mathematics. Springer Verlag, second edition, 2010.

34. Ellis Robert Kolchin. Differential Algebra and Algebraic Groups. Academic Press, New York, 1973.

35. Vladimir Alexandrovitch Kostitzin. Biologie Mathématique. Armand Colin, 1937. (avec une préface de Vito Volterra).

36. Markus Lange-Hegermann and Daniel Robertz. Thomas decompositions of parametric nonlinear control systems. IFAC Proceedings Volumes, 46(2):296-301, 2013.

37. L. Ljung and S. T. Glad. On global identifiability for arbitrary model parametrisations. Automatica, 30:265-276, 1994.

38. Jacob Lubliner. Plasticity Theory. Dover, 2008.

39. Elizabeth L. Mansfield. Differential Gröbner Bases. PhD thesis, University of Sydney, Australia, 1991.

40. Mamadou Mboup, Cédric Join, and Michel Fliess. Numerical differentiation with annihilator in noisy environment. Numerical algorithm, 50(4):439-467, 2009.

41. Céline Noiret. Utilisation du calcul formel pour l'identifiabilité de modèles paramétriques et nouveaux algorithmes en estimation de paramètres. $\mathrm{PhD}$ thesis, Université de Technologie de Compiègne, 2000. 
42. François Ollivier. Le problème de l'identifiabilité structurelle globale : approche théorique, méthodes effectives et bornes de complexité. $\mathrm{PhD}$ thesis, École Polytechnique, Palaiseau, France, 1990.

43. Alain Pavé. Modeling living systems, from cell to ecosystem. ISTE/Wiley, 2012.

44. Émile Picard. La mathématique dans ses rapports avec la physique. In Actes du IVème congrès international des mathématiques, Rome, Italie, 1908. Gauthier-Villars. 10 avril 1908.

45. Alban Quadrat and Georg Regensburger. Polynomial solutions and annihilators of ordinary integro-differential operators. IFAC Proceedings Volumes, 46(2):308-313, 2013.

46. Nicolas Rashevsky. Mathematical Biophysics: Physico-Mathematical Foundations of Biology. Vol. 1. Dover Publ. Inc., third edition, 1960.

47. Gregory J. Reid, Allan D. Wittkopf, and Alan Boulton. Reduction of systems of nonlinear partial differential equations to simplified involutive forms. European Journal of Applied Math., 7(6):635-666, 1996.

48. Joseph Fels Ritt. Differential Algebra, volume 33 of American Mathematical Society Colloquium Publications. American Mathematical Society, New York, 1950.

49. Daniel Robertz. Formal Algorithmic Elimination for PDEs, volume 2121 of Lecture Notes in Mathematics. Springer Verlag, 2014.

50. Markus Rosenkranz. A new symbolic method for solving linear two-point boundary value problems on the level of operators. J. Symbolic Comput., 39(2):171-199, 2005.

51. Markus Rosenkranz, Xing Gao, and Li Guo. An Algebraic Study of Multivariable Integration and Linear Substitution. Technical Report 1503.01694, arXiv, 2015.

52. Markus Rosenkranz and Georg Regensburger. Integro-Differential Polynomials and Operators. In David Jeffrey, editor, ISSAC'08: Proceedings of the 2008 International Symposium on Symbolic and Algebraic Computation. ACM Press, 2008.

53. Markus Rosenkranz and Georg Regensburger. Solving and Factoring Boundary Problems for Linear Ordinary Differential Equations in Differential Algebras. Journal of Symbolic Computation, 43(8):515-544, 2008.

54. Gian-Carlo Rota. Baxter algebras and combinatorial identities (I, II). Bull. Amer. Math. Soc., 75:325-334, 1969.

55. Gian-Carlo Rota. Baxter operators, an introduction. In Gian-Carlo Rota on Combinatorics, Introductory papers and Commentaries. Birkhäuser, Boston, 1995.

56. Alexandre Sedoglavic. A Probabilistic Algorithm to Test Local Algebraic Observability in Polynomial Time. Journal of Symb. Comp., 33(5):735-755, 2002.

57. Abraham Seidenberg. An elimination theory for differential algebra. Univ. California Publ. Math. (New Series), 3:31-65, 1956.

58. Nathalie Verdière, Lilianne Denis-Vidal, and Ghislaine Joly-Blanchard. A new method for estimating derivatives based on a distribution approach. Numerical Algorithms, 61:163-186, 2012.

59. Nathalie Verdière, Lilianne Denis-Vidal, Ghislaine Joly-Blanchard, and Dominique Domurado. Identifiability and Estimation of Pharmacokinetic Parameters for the Ligands of the Macrophage Mannose Receptor. Int. J. Appl. Math. Comput. Sci., 15(4):517-526, 2005.

60. Vito Volterra. Sur les équations intégro-différentielles et leurs applications. Acta Mathematica, 35(1):295-356, 1912.

61. Vito Volterra. Leçons sur les équations intégrales et les équations intégro-différentielles. Gauthier-Villars, Paris, 1913. Leçons professées à la faculté des sciences de Rome, publiées par M. Tomassetti et F.-S. Zarlatti.

62. Vito Volterra. Applications des mathématiques à la biologie. L'enseignement mathématique, 1937. Leçon faite le 17 juin 1937, dans la série des Conférences internationales des Sciences mathématiques.

63. Vito Volterra. Theory of functionals and of integral and integro-differential equations. Dover Publ. Inc., 1959. with a biography and a bibliography by Sir Edmund Whittaker. 